\title{
Packing densities of layered permutations and the minimum number of monotone sequences in layered permutations
}

\author{
Josefran de Oliveira Bastos* $\quad$ Leonardo Nagami Coregliano $^{\dagger}$ \\ Instituto de Matemática e Estatística, Universidade de São Paulo, Brazil
}

received $28^{\text {th }}$ Oct. 2015, revised $14^{\text {th }}$ June 2016, accepted $17^{\text {th }}$ June 2016.

In this paper, we present two new results of layered permutation densities. The first one generalizes theorems from Hästö (2003) and Warren (2004) to compute the permutation packing of permutations whose layer sequence is $\left(1^{a}, \ell_{1}, \ell_{2}, \ldots, \ell_{k}\right)$ with $2^{a}-a-1 \geq k$ (and similar permutations). As a second result, we prove that the minimum density of monotone sequences of length $k+1$ in an arbitrarily large layered permutation is asymptotically $1 / k^{k}$. This value is compatible with a conjecture from Myers (2003) for the problem without the layered restriction (the same problem where the monotone sequences have different lengths is also studied).

Keywords: permutations, packing densities, extremal combinatorics, monotone subsequences

\section{Introduction}

As usual, a permutation over $[n]=\{1,2, \ldots, n\}$ is a bijective function of $[n]$ onto itself. We denote the set of all permutations over $[n]$ by $\mathfrak{S}_{n}$ and, for every $\sigma \in \mathfrak{S}_{n}$, we say that the length of $\sigma$ (denoted $|\sigma|$ ) is $n$.

We denote by $\mathbb{N}$ the set of non-negative integers and let $\mathbb{N}^{*}=\mathbb{N} \backslash\{0\}$. We also denote by $\mathfrak{S}=\bigcup_{n \in \mathbb{N}} \mathfrak{S}_{n}$ the set of all finite permutations.

We also use the notation $\left\{i_{1}, i_{2}, \ldots, i_{k}\right\}<\subset A$ as a shorthand for $\left\{i_{1}, i_{2}, \ldots, i_{k}\right\} \subset A$ with $i_{1}<i_{2}<$ $\cdots<i_{k}$. Furthermore, we frequently denote a permutation $\sigma \in \mathfrak{S}_{n}$ by $(\sigma(1) \sigma(2) \cdots \sigma(n))$ using extra parentheses whenever the notation starts to get too ambiguous.

Let $\sigma \in \mathfrak{S}_{n}$ and let $\left\{i_{1}, i_{2}, \ldots, i_{m}\right\}<\subset[n]$, the subpermutation induced by $\left\{i_{1}, i_{2}, \ldots, i_{m}\right\}$ in $\sigma$ is the unique permutation $\tau \in \mathfrak{S}_{m}$ such that for every $j, k \in[m]$ we have $\sigma\left(i_{j}\right)<\sigma\left(i_{k}\right)$ if and only if $\tau(j)<\tau(k)$ and it is denoted by $\sigma\left[\left\{i_{1}, i_{2}, \ldots, i_{m}\right\}\right]$. For example, if $\sigma=(68153427)$, then we have $\sigma[\{1,3,6\}]=(312)$ and $\sigma[\{2,4,7,8\}]=(4213)$.

\footnotetext{
* Supported by Coordenação de Aperfeiçoamento de Pessoal de Nível Superior (CAPES/Brazil).

${ }^{\dagger}$ Supported by São Paulo Research Foundation (FAPESP), grant \#2013/23720-9.
} 
Furthermore, if $\tau \in \mathfrak{S}_{m}$ and $\sigma \in \mathfrak{S}_{n}$, then we define the number of occurrences $\Lambda(\tau, \sigma)$ of the permutation $\tau$ in $\sigma$ as

$$
\Lambda(\tau, \sigma)=|\{A \subset[n]: \sigma[A]=\tau\}|=\left|\left\{A \in\left(\begin{array}{c}
{[n]} \\
m
\end{array}\right): \sigma[A]=\tau\right\}\right|,
$$

where

$$
\left(\begin{array}{c}
{[n]} \\
m
\end{array}\right)=\{A \subset[n]:|A|=m\} .
$$

We also define the density $p(\tau, \sigma)$ of $\tau$ in $\sigma$ as

$$
p(\tau, \sigma)= \begin{cases}\left(\begin{array}{c}
n \\
m
\end{array}\right)^{-1} \Lambda(\tau, \sigma), & \text { if } m \leq n \\
0, & \text { if } m>n\end{cases}
$$

which, when $m \leq n$, coincides with the probability that we induce $\tau$ by picking uniformly at random an element of $\left(\begin{array}{c}{[n]} \\ m\end{array}\right)=\{A \subset[n]:|A|=m\}$. We extend the definitions of $\Lambda$ and $p$ linearly in the first coordinate to (finite) formal linear combinations of elements of $\mathfrak{S}$ (i.e., we extend their domain to $\mathbb{R} \mathfrak{S} \times \mathfrak{S}$ ). Let $f \in \mathbb{R} \mathfrak{S}$ be a formal linear combination of permutations. For every $N \in \mathbb{N}$, define

$$
\begin{aligned}
p_{N}(f) & =\max \left\{p(f, \sigma): \sigma \in \mathfrak{S}_{N}\right\} ; \\
\operatorname{Ext}_{N}(f) & =\left\{\sigma \in \mathfrak{S}_{N}: p(f, \sigma)=p_{N}(f)\right\} ; \\
p(f) & =\lim _{N \rightarrow \infty} p_{N}(f) .
\end{aligned}
$$

The following argument, which has already become part of the folklore of extremal combinatorics, proves that the above limit indeed exists.

Lemma 1.1. For every $f \in \mathbb{R S}$, there exists $N_{0} \in \mathbb{N}$ such that $p_{N}(f) \geq p_{N+1}(f)$ for every $N \geq N_{0}$.

Proof: Write $f=\sum_{i=1}^{k} c_{i} \tau_{i}$ and let $N_{0}=\max \left\{\left|\tau_{i}\right|: i \in[k]\right\}$.

Note that if $N \geq N_{0}$ and $\sigma \in \mathfrak{S}_{N+1}$ is such that $p_{N+1}(f)=p(f, \sigma)$, then we can compute $p\left(\tau_{i}, \sigma\right)$ using the following random experiment. We first pick $j \in[N+1]$ uniformly at random then we compute the density of $\tau_{i}$ in $\sigma[[N+1] \backslash\{\boldsymbol{j}\}]$. Note that

$$
p\left(\tau_{i}, \sigma\right)=\mathbb{E}\left[p\left(\tau_{i}, \sigma[[N+1] \backslash\{\boldsymbol{j}\}]\right)\right] .
$$

But then, by linearity of expectation, we have

$$
p_{N+1}(f)=p(f, \sigma)=\mathbb{E}[p(f, \sigma[[N+1] \backslash\{\boldsymbol{j}\}])] \leq p_{N}(f),
$$

as desired.

One of the most studied problems involving permutations is the packing density problem stated below.

Problem 1.2 (Permutation packing). Let $f \in \mathbb{R} \mathfrak{S}$ be a formal linear combination of permutations. For every $N \in \mathbb{N}$, how large can $p(f, \sigma)$ be and what are the properties that a $\sigma \in \operatorname{Ext}_{N}(f)$ has? 
In this problem, one interesting subfamily of $\mathfrak{S}$ is the family of layered permutations. A permutation $\sigma \in \mathfrak{S}_{n}$ of length $n$ is called layered if there exists $\left\{i_{1}, i_{2}, \ldots, i_{k}\right\}<\subset[n+1]$ with $i_{1}=1$ and $i_{k}=n+1$ such that

$$
\sigma\left[\left\{i_{j}, i_{j}+1, \ldots, i_{j+1}-1\right\}\right]=\left(\left(i_{j+1}-1\right)\left(i_{j+1}-2\right) \cdots\left(i_{j}+1\right) i_{j}\right),
$$

for every $j \in[k-1]$, and $\sigma(a)<\sigma(b)$ whenever $a, b \in[n]$ are such that $a<i_{j} \leq b$ for some $j \in[k-1]$.

This means that the permutation $\sigma$ consists of an increasing sequence of decreasing sequences. Such decreasing sequences are called layers of $\sigma$. We denote a layered permutation by the sequence of lengths of its layers as $\left(\ell_{1}, \ell_{2}, \ldots, \ell_{k}\right)$ (it is easy to see that such representation is unique) and we call such a sequence the layer sequence or layer decomposition of $\sigma$.

Note that the function that maps a layered permutation to its layer sequence provides an isomorphism to the theory of compositions (i.e., ordered partitions of $n$ ).

An antilayer of a layered permutation $\sigma$ is a maximal contiguous non-empty sequence of layers of length 1. A block of a layered permutation is either a layer of length at least 2 or an antilayer, i.e., a block is a maximal monotone interval. When it is convenient, we also denote a layered permutation by the sequence of lengths of its blocks using a "hat" to denote when the corresponding block is an antilayer, and we call this sequence, the block sequence or the block decomposition of the permutation, e.g., the permutation $\sigma=(321457689)$ has block decomposition $(3, \widehat{2}, 2, \widehat{2})$. The theorem below shows that the problem of packing a layered permutation is much easier than the general case.

Throughout this paper, we let $\mathbb{R} \mathfrak{S}$ denote the set of formal linear combinations of elements of $\mathfrak{S}$ with real coefficients and we say that $f \in \mathbb{R} \mathfrak{S}$ is a conical combination when all its coefficients are nonnegative.

Theorem 1.3 (Albert et al. (2002)). If $f \in \mathbb{R S}$ is a conical combination of layered permutations then, for every $N \in \mathbb{N}$, there exists a layered permutation in $\operatorname{Ext}_{N}(f)$. This in particular means that

$$
p_{N}(f)=\max \left\{p(f, \sigma): \sigma \in \mathfrak{S}_{N} \text { is layered }\right\} .
$$

Based on the theorem above, Price (1997) suggested an algorithm that, given a conical combination $f$ of layered permutations, computes lower bounds to $p(f)$ converging to $p(f)$.

Much more work has been done to compute the packing density problem of layered permutations than of non-layered permutations. Albert et al. (2002) solved the problem of layered permutations with two layers. Hästö (2002/03) solved the problem for any layered permutation that has $r$ layers of length at least $\log r+1$. He also solved the problem for all layered permutations of the form $(k, 1, k)$ with $k \geq 3$. Our first result in this work is the following.

Theorem 1.4. Let $a, \ell_{1}, \ell_{2}, \ldots, \ell_{k} \in \mathbb{N}^{*}$ be positive integers such that $2 \leq a \leq \ell_{1} \leq \ell_{2} \leq \cdots \leq \ell_{k}$ and $2^{a}-a-1 \geq k$. If $\sigma$ is the layered permutation $\left(\widehat{a}, \ell_{1}, \ell_{2}, \ldots, \ell_{k}\right)$, then we have

$$
p(\sigma)=\frac{|\sigma| !}{|\sigma|^{|\sigma|}} \frac{a^{a}}{a !} \prod_{i=1}^{k} \frac{\ell_{i}^{\ell_{i}}}{\ell_{i} !} .
$$

The packing density problem is focused on estimating the permutation that maximizes the number of occurrences of a smaller permutation. It is natural to think of the dual problem, i.e., trying to find the permutation that minimizes the number of occurrences of a smaller permutation. 
Problem 1.5 (Permutation minimization). Let $f \in \mathbb{R S}$ be a formal linear combination of permutations. For every $N \in \mathbb{N}$, define

$$
\begin{aligned}
p_{N}^{\prime}(f) & =\min \left\{p(f, \sigma): \sigma \in \mathfrak{S}_{N}\right\} ; \\
\operatorname{Ext}_{N}^{\prime}(f) & =\left\{\sigma \in \mathfrak{S}_{N}: p(f, \sigma)=p_{N}^{\prime}(f)\right\} ; \\
p^{\prime}(f) & =\lim _{N \rightarrow \infty} p_{N}^{\prime}(f) .
\end{aligned}
$$

The problem then consists of computing explicitly the values above, which basically means answering the question: how small can $p(f, \sigma)$ be and what are the properties that a $\sigma \in \operatorname{Ext}_{N}^{\prime}(f)$ has?

Although we can restate the minimization problem as a permutation packing problem, we lose the result from Theorem 1.3 by doing so. So the next problem is a completely different problem.

Problem 1.6 (Layered permutation minimization). Let $f \in \mathbb{R} S$ be a formal linear combination of layered permutations. For every $N \in \mathbb{N}$, define

$$
\begin{aligned}
p_{N}^{\prime \prime}(f) & =\min \left\{p(f, \sigma): \sigma \in \mathfrak{S}_{N} \text { and } \sigma \text { is layered }\right\} \\
\operatorname{Ext}_{N}^{\prime \prime}(f) & =\left\{\sigma \in \mathfrak{S}_{N} \text { layered }: p(f, \sigma)=p_{N}^{\prime \prime}(f)\right\} ; \\
p^{\prime \prime}(f) & =\lim _{N \rightarrow \infty} p_{N}^{\prime \prime}(f) .
\end{aligned}
$$

The problem then consists of computing explicitly the values above, which basically means answering the question: how small can $p(f, \sigma)$ be if $\sigma$ is layered and what are the properties that a $\sigma \in \operatorname{Ext}_{N}^{\prime \prime}(f)$ has?

For every length $n \in \mathbb{N}$, two particular permutations deserve special notation. One is the identity, denoted by $\operatorname{Id}_{n}=(12 \cdots n)$, and the other is the reverse, denoted by $\operatorname{Rev}_{n}=(n(n-1) \cdots 1)$.

A well-known theorem by Erdös and Szekeres (1935) states that every permutation of $k^{2}+1$ elements must contain a monotone subsequence of length $k+1$. Later, Myers proved the following quantitative version of this theorem.

Theorem 1.7 (Myers (2002/03)). We have

$$
p^{\prime}\left(\operatorname{Id}_{3}+\operatorname{Rev}_{3}\right)=\frac{1}{4}
$$

Furthermore, for every $k \geq 2$, we have

$$
\lim _{N \rightarrow \infty} \min \left\{p\left(\operatorname{Rev}_{k+1}, \sigma\right): \sigma \in \mathfrak{S}_{N} \text { with } p\left(\operatorname{Id}_{k+1}, \sigma\right)=0\right\}=\frac{1}{k^{k}} .
$$

The second part of the theorem above led Myers to conjecture that $p^{\prime}\left(\operatorname{Id}_{k+1}+\operatorname{Rev}_{k+1}\right)=1 / k^{k}$ for every $k \geq 2$ (the case $k=2$ is the first part of the theorem). We state a generalized version of Myers' Conjecture below.

Conjecture 1.8 (Generalization of Myers (2002/03)). For every $k, \ell \geq 2$, we have

$$
p^{\prime}\left(\operatorname{Id}_{\ell+1}+\operatorname{Rev}_{k+1}\right)=\min \left\{\frac{1}{k^{\ell}}, \frac{1}{\ell^{k}}\right\} .
$$


The minimum above is actually an upper bound to $p^{\prime}\left(\operatorname{Id}_{\ell+1}+\operatorname{Rev}_{k+1}\right)$, obtained by considering permutations that have only one of the monotone sequences counted.

Using the tool of Flag Algebras developed by Razborov (2007), the case $k=\ell=3$ was proved to be true by Balogh et al. (2013).

We also remark that Samotij and Sudakov (2015) proved a version of this conjecture when $n \leq k^{2}+$ $c k^{3 / 2} / \log k$ and $k$ and $c$ are sufficiently large.

As our second result, we solve the simpler version of this problem when we restrict ourselves to the class of layered permutations, that is, we compute $p^{\prime \prime}\left(\operatorname{Id}_{\ell+1}+\operatorname{Rev}_{k+1}\right)$.

Theorem 1.9. If $k \geq \ell \geq 3$, then we have $p^{\prime \prime}\left(\operatorname{Id}_{\ell}+\operatorname{Rev}_{k}\right)=1 /(\ell-1)^{k-1}$. In particular, for $m \geq 2$, we have $p^{\prime \prime}\left(\operatorname{Id}_{m+1}+\operatorname{Rev}_{m+1}\right)=1 / m^{m}$.

The paper is organized as follows. In Section 2, we present Price's Algorithm, one generalization of it, and the first result, which concerns permutation packing. In Section 3, we present the second result, which concerns minimization of the asymptotic density of monotone sequences in layered permutations. Finally, in Section 4, we conclude the text by presenting some related work and conjectures.

\section{Generalizing Price's Algorithm}

We start by presenting the original Price's Algorithm to compute a lower bound for the packing density of a layered permutation. The idea behind the algorithm is to note that the density of a layered permutation in an arbitrarily large layered permutation with a bounded number of layers can be expressed by a polynomial.

Let $\tau \in \mathfrak{S}_{m}$ be a layered permutation with layer sequence $\left(\ell_{j}\right)_{j=1}^{k}$. We define the Price Polynomial of order $n \in \mathbb{N}^{*}$ for $\tau$ to be the real polynomial over $n$ variables given by

$$
q_{n, \tau}\left(x_{1}, x_{2}, \ldots, x_{n}\right)=m ! \sum_{\left\{i_{1}, i_{2}, \ldots, i_{k}\right\}<\subset[n]} \prod_{j=1}^{k} \frac{x_{i_{j}}^{\ell_{j}}}{\ell_{j} !}=\left(\begin{array}{c}
m \\
\ell_{1}, \ell_{2}, \ldots, \ell_{k}
\end{array}\right) \sum_{\left\{i_{1}, i_{2}, \ldots, i_{k}\right\}<\subset[n]} \prod_{j=1}^{k} x_{i_{j}}^{\ell_{j}},
$$

where an empty sum is taken to have result 0 and an empty product is taken to have result 1 .

We also define the Price Polynomial of order $n \in \mathbb{N}^{*}$ for a linear combination of layered permutations $f=\sum_{i=1}^{k} a_{i} \tau_{i}$ as

$$
q_{n, f} \equiv \sum_{i=1}^{k} a_{i} q_{n, \tau_{i}}
$$

The Price Bound of order $n \in \mathbb{N}^{*}$ for a linear combination of layered permutations $f$ is the value

$$
\mathfrak{L}_{n, f}=\max \left\{q_{n, f}\left(x_{1}, x_{2}, \ldots, x_{n}\right): \sum_{j=1}^{n} x_{j}=1 \text { and } \forall j \in[n], x_{j} \geq 0\right\} .
$$

Remark. This maximum exists since the set $\left\{\left(x_{1}, x_{2}, \ldots, x_{n}\right) \in[0,1]^{n}: \sum_{j=1}^{n} x_{j}=1\right\}$ is compact and $q_{n, f}$ is continuous. 
Proposition 2.1 (Price (1997)). If $f \in \mathbb{R} \mathfrak{S}$ is a conical combination of layered permutations, then, for every $n \in \mathbb{N}^{*}$, we have $\mathfrak{L}_{n, f} \leq \mathfrak{L}_{n+1, f} \leq p(f)$.

The proposition above suggests a simple algorithm of computing approximations for the values $\mathfrak{L}_{n, f}$ (which are maxima of polynomials) to get lower bounds for $p(f)$. This is why this is called "Price's Algorithm".

Corollary 2.2 (of Theorem 1.3 and Proposition 2.1, Price (1997)). If $f \in \mathbb{R} S$ is a conical combination of layered permutations, then we have

$$
\lim _{n \rightarrow \infty} \mathfrak{L}_{n, f}=p(f) .
$$

We now present some definitions that will be useful for generalizing Price's Algorithm to consider antilayers.

Recall that an antilayer is a maximal contiguous non-empty sequence of layers of length 1 and that a block is either a layer of length at least 2 or an antilayer. We formalize the definition of the block sequence of a layered permutation $\sigma$ as the unique sequence $\left(\ell_{i}, \xi_{i}\right)_{i=1}^{k}$ of elements of $\mathbb{N}^{*} \times\{0,1\}$ such that, for every $i \in[k]$, we have

$$
\left\{\sum_{j=1}^{i-1} \ell_{j}+t: t \in\left[\ell_{i}\right]\right\} \text { is } \begin{cases}\text { a layer and } \ell_{i} \geq 2, & \text { if } \xi_{i}=0 \\ \text { an antilayer, } & \text { if } \xi_{i}=1 .\end{cases}
$$

In other words, the value $\xi_{i}=1$ corresponds to the "hat" in the notation of block sequence defined previously.

We will also need some slightly different notions from the above. An antilayeroid of a layered permutation $\sigma$ is a contiguous non-empty subsequence of an antilayer. A quasi-block of a layered permutation $\sigma$ is either a layer of length at least 2 or an antilayeroid. A quasi-block sequence or quasi-block decomposition of a layered permutation $\sigma$ is a sequence $\left(\ell_{i}, \xi_{i}\right)_{i=1}^{k}$ of elements of $\mathbb{N}^{*} \times\{0,1\}$ such that, for every $i \in[k]$, we have

$$
\left\{\sum_{j=1}^{i-1} \ell_{j}+t: t \in\left[l_{i}\right]\right\} \text { is } \begin{cases}\text { a layer and } \ell_{i} \geq 2, & \text { if } \xi_{i}=0 \\ \text { an antilayeroid, } & \text { if } \xi_{i}=1 .\end{cases}
$$

Informally, a quasi-block decomposition of $\sigma$ is obtained from the block decomposition by splitting antilayers of $\sigma$ into any number of (pairwise disjoint) antilayeroids.

Note that, in the quasi-block decomposition, we can have two consecutive antilayeroids (the analogous situation for block decompositions never happens).

We extend the "hat" notation of block decompositions to quasi-block decompositions by using a "hat" to indicate which quasi-blocks are antilayeroids.

We denote the set of quasi-block decompositions of a layered permutation by $\mathfrak{Q}_{\sigma}$.

As an example, for $\sigma=(321457689)$, we have

$$
\mathfrak{Q}_{\sigma}=\{(3, \widehat{2}, 2, \widehat{2}),(3, \widehat{2}, 2, \widehat{1}, \widehat{1}),(3, \widehat{1}, \widehat{1}, 2, \widehat{2}),(3, \widehat{1}, \widehat{1}, 2, \widehat{1}, \widehat{1})\} .
$$

Note that, the block decomposition of $\sigma$ is also a quasi-block decomposition of $\sigma$. Note also that quasi-block decompositions are not unique (unless every antilayer has length 1 ). 
The usefulness of this decomposition arises when we consider an occurrence of a layered permutation $\tau$ in another layered permutation $\sigma$, because if two points of $\tau$ occur in the same block of $\sigma$, then there must be a quasi-block of $\tau$ that has both points (note that this is no longer true if we replace quasi-block by block since it is possible to split an antilayer of $\tau$ to fit into two or more blocks of $\sigma$ ). Within this context of occurrences, we can define the concept of natural decomposition as follows.

Let $A=\left\{i_{1}, i_{2}, \ldots, i_{n}\right\}<$ be an occurrence of a layered permutation $\tau$ in another layered permutation $\sigma$. The natural decomposition induced by $A$ and $\sigma$ in $\tau$ is the unique quasi-block decomposition (denoted by $\mathfrak{N}(A, \sigma)$ ) of $\tau$ such that points of $\tau$ are in the same quasi-block of $\mathfrak{N}(A, \sigma)$ if and only if the corresponding indices of $A$ in the same block of $\sigma$.

As an example, if $\sigma=(21346587), \tau=(21345)$, and $A=\{1,2,3,4,8\}$, then $\mathfrak{N}(A, \sigma)=(2, \widehat{2}, \widehat{1})$.

An easy way to obtain the natural decomposition of $\tau$ from its occurrence $A$ in $\sigma$ and the block decomposition $\left(\ell_{i}^{\prime}, \xi_{i}^{\prime}\right)_{i=1}^{k}$ of $\sigma$ is to first take $\ell_{i}$ as the number of points of $A$ in the $i$-th block of $\sigma$ and $\xi_{i}=\xi_{i}^{\prime}$ and then remove any zero-length quasi-blocks that arose in the process and change any $\xi_{i}$ of the layers of length 1 to 1 .

In the example given, this process yields

$$
(2, \widehat{2}, 2,2) \longrightarrow(2, \widehat{2}, 0,1) \longrightarrow(2, \widehat{2}, \widehat{1}) .
$$

The uniqueness of natural decompositions allows us to partition the occurrences according to the natural decompositions that they induce.

Although "Price's Algorithm" always converges to the packing density, when we have a layered permutation with an antilayer, the natural way to fit it in another layered permutation is to put it in another antilayer. Since "Price's Algorithm" uses only layers, if the extremal permutation has an antilayer, the algorithm will gradually produce it through a sequence of small variables. With this in mind, we generalize "Price's Algorithm" to consider antilayers. The intuition is to construct polynomials analogous to Price Polynomials but alternating antilayers and layers in the "arbitrarily large permutation" representing the polynomial (i.e., variables with odd subscripts correspond to antilayers and variables with even subscripts correspond to layers).

Definition 2.3. Let $\tau \in \mathfrak{S}_{m}$ be a layered permutation of length $m$.

We define the Extended Price Polynomial of order $n \in \mathbb{N}^{*}$ for $\tau$ to be the real polynomial over $2 n$ variables given by

$$
g_{n, \tau}\left(x_{1}, x_{2}, \ldots, x_{2 n}\right)=m ! \sum_{\left(\ell_{j}, \xi_{j}\right)_{j=1}^{k} \in \mathfrak{Q}_{\tau}} \sum_{\left\{i_{1}, i_{2}, \ldots, i_{k}\right\}<\subset[2 n]} \prod_{j=1}^{k} \frac{x_{i_{j}}^{\ell_{j}}}{\ell_{j} !} \mathbb{1}_{\left\{i_{j} \bmod 2=\xi_{j} \text { or } \ell_{j}=1\right\}},
$$

where $\mathbb{1}$ denotes the indicator function.

We also define the Price Polynomial of order $n \in \mathbb{N}^{*}$ for a linear combination of layered permutations $f=\sum_{i=1}^{k} a_{i} \tau_{i}$ as

$$
g_{n, f} \equiv \sum_{i=1}^{k} a_{i} g_{n, \tau_{i}}
$$


Note that the indicator function is responsible for making sure that variables with odd subscripts correspond to antilayers (which can contain only antilayeroids of $\tau$ ) and that variables with even subscripts correspond to layers (which can contain only layers of $\tau$ ).

The Extended Price Bound defined below has yet another parameter which is responsible for forcing some of the antilayers of the "arbitrarily large permutation" to have length zero.

Definition 2.4. Let $f \in \mathbb{R S}$ be a linear combination of layered permutations, let $n \in \mathbb{N}^{*}$ be a positive integer, and let $W \subset[n]$.

The Extended Price Bound of order $n$ relative to $W$ for $f$ is the value

$$
\mathfrak{L}_{n, W, f}=\max \left\{g_{n, f}\left(x_{1}, x_{2}, \ldots, x_{2 n}\right): \sum_{j=1}^{2 n} x_{j}=1 \text { and } \forall j \in[n], x_{j} \geq 0 \text { and } \forall j \in W, x_{2 j-1}=0\right\} .
$$

Remark. Once again this maximum exists by a compactness argument. Furthermore, note that $\mathfrak{L}_{n, f}=$ $\mathfrak{L}_{n,[n], f}$.

Let us now prove the convergence of the analogous "Extended Price's Algorithm".

Theorem 2.5. If $f=\sum_{t=1}^{k} a_{t} \tau_{t} \in \mathbb{R S}$ is a conical combination of layered permutations and $\left(W_{n}\right)_{n \in \mathbb{N} *}$ is a sequence of sets such that $W_{n} \subset[n]$ for every $n \in \mathbb{N}^{*}$, then we have

$$
\lim _{n \rightarrow \infty} \mathfrak{L}_{n, W_{n}, f}=p(f) .
$$

Proof: Note first that if $x_{1}, x_{2}, \ldots, x_{n} \geq 0$ are such that $\sum_{j=1}^{n} x_{j}=1$ and $q_{n, f}\left(x_{1}, x_{2}, \ldots, x_{n}\right)=\mathfrak{L}_{n, f}$, then we have

$$
\mathfrak{L}_{n, W_{n}, f} \geq g_{n, f}\left(0, x_{1}, 0, x_{2}, \ldots, 0, x_{n}\right)=q_{n, f}\left(x_{1}, x_{2}, \ldots, x_{n}\right)=\mathfrak{L}_{n, f} .
$$

Therefore, by Corollary 2.2, we have $\lim _{\inf _{n \rightarrow \infty}} \mathfrak{L}_{n, W_{n}, f} \geq p(f)$.

Now let us prove the other inequality with $\lim \sup$. Fix $n \in \mathbb{N}^{*}$ and let us prove that $\mathfrak{L}_{n, W_{n}, f} \leq p(f)$.

Let $\epsilon>0$ be an arbitrary positive real number and $b_{1}, b_{2}, \ldots, b_{2 n}, N \in \mathbb{N}$ with $N \neq 0$ be such that $\sum_{j=1}^{2 n} b_{j}=N, b_{2 j-1}=0$ for every $j \in W_{n}, b_{2 j} \neq 0$ for every $j \in[n]$ and

$$
g_{n, f}\left(\frac{b_{1}}{N}, \frac{b_{2}}{N}, \ldots, \frac{b_{2 n}}{N}\right) \geq \mathfrak{L}_{n, W_{n}, f}-\epsilon .
$$

Now, for every $m \in \mathbb{N}^{*}$, let $\sigma_{m} \in \mathfrak{S}_{m N}$ be the layered permutation of length $m N$ and with block sequence $\left(\widehat{m b_{1}}, m b_{2}, \widehat{m b_{3}}, m b_{4}, \ldots, \widehat{m b_{2 n-1}}, m b_{2 n}\right)$ (if any of these numbers is zero, we remove it from the sequence to form $\sigma_{m}$ ).

Now, for every $t \in[k]$, we count the occurrences of $\tau_{t}$ in $\sigma_{m}$ according to the natural decomposition 
that they induce in $\tau_{t}$. So we have

$$
\begin{aligned}
p\left(\tau_{t}, \sigma_{m}\right) & =\left(\begin{array}{c}
m N \\
\left|\tau_{t}\right|
\end{array}\right)^{-1} \sum_{\left(\ell_{j}, \xi_{j}\right)_{j=1}^{k} \in \mathfrak{Q}_{\tau_{t}}} \sum_{\left\{i_{1}, \ldots, i_{k}\right\}<\subset[2 n]} \prod_{j=1}^{k}\left(\begin{array}{c}
m b_{i_{j}} \\
\ell_{j}
\end{array}\right) \mathbb{1}_{\left\{i_{j} \bmod 2=\xi_{j} \text { or } \ell_{j}=1\right\}} \\
& \sim \frac{\left|\tau_{t}\right| !}{(m N)^{\left|\tau_{t}\right|}} \sum_{\left(\ell_{j}, \xi_{j}\right)_{j=1}^{k} \in \mathfrak{Q}_{\tau_{t}}} \sum_{\left\{i_{1}, \ldots, i_{k}\right\}<\subset[2 n]} \prod_{j=1}^{k} \frac{\left(m b_{i_{j}}\right)^{\ell_{j}}}{\ell_{j} !} \mathbb{1}_{\left\{i_{j} \bmod 2=\xi_{j} \text { or } \ell_{j}=1\right\}} \\
& =\left|\tau_{t}\right| ! \sum_{\left(\ell_{j}, \xi_{j}\right)_{j=1}^{k} \in \mathfrak{Q}_{\tau_{t}}} \sum_{\left\{i_{1}, \ldots, i_{k}\right\}<\subset[2 n]} \prod_{j=1}^{k} \frac{\left(b_{i_{j}} / N\right)^{\ell_{j}}}{\ell_{j} !} \mathbb{1}_{\left\{i_{j} \bmod 2=\xi_{j} \text { or } \ell_{j}=1\right\} .} \\
& =g_{n, \tau_{t}}\left(\frac{b_{1}}{N}, \frac{b_{2}}{N}, \ldots, \frac{b_{2 n}}{N}\right),
\end{aligned}
$$

where $\sim$ means that the ratio between both sides goes to 1 as $m$ goes to $\infty$ and the first equality after $\sim$ follows from the fact that $\sum_{j=1}^{k} \ell_{j}=\left|\tau_{t}\right|$ for every quasi-block decomposition $\left(\ell_{j}, \xi_{j}\right)_{j=1}^{k}$ of $\tau_{t}$.

Therefore, we have

$$
\begin{aligned}
p\left(f, \sigma_{m}\right) & =\sum_{t=1}^{k} a_{t} p\left(\tau_{t}, \sigma_{m}\right) \\
& \sim \sum_{t=1}^{k} a_{t} g_{n, \tau_{t}}\left(\frac{b_{1}}{N}, \frac{b_{2}}{N}, \ldots, \frac{b_{2 n}}{N}\right) \\
& =g_{n, f}\left(\frac{b_{1}}{N}, \frac{b_{2}}{N}, \ldots, \frac{b_{2 n}}{N}\right) \\
& \geq \mathfrak{L}_{n, W_{n}, f}-\epsilon .
\end{aligned}
$$

So, for every $\epsilon>0$, we have

$$
p(f) \geq \lim _{m \rightarrow \infty} p\left(f, \sigma_{m}\right) \geq \mathfrak{L}_{n, W_{n}, f}-\epsilon .
$$

Since $\epsilon$ is arbitrary, we have $\mathfrak{L}_{n, W_{n}, f} \leq p(f)$ for every $n \in \mathbb{N}^{*}$, hence

$$
p(f) \geq \limsup _{n \rightarrow \infty} \mathfrak{L}_{n, W_{n}, f} .
$$

Therefore $\lim _{n \rightarrow \infty} \mathfrak{L}_{n, W_{n}, f}=p(f)$.

\subsection{Using generalizations}

We now introduce some notation to help in the proof of Theorem 1.4.

Notation 2.6. Let $T \subset \mathbb{Z}$ be a set of integers and $\left(x_{t}\right)_{t \in T}$ be a sequence indexed by elements of $T$. Let also $I=\left\{i_{1}, \ldots, i_{k}\right\}<\subset T$ be a subset of $T$. We denote by $x_{I}$ the (ordered) sequence $\left(x_{i_{1}}, \ldots, x_{i_{k}}\right)$. 
Furthermore, if $x=\left(x_{1}, x_{2}, \ldots, x_{k}\right)$ and $y=\left(y_{1}, y_{2}, \ldots, y_{k}\right)$ are sequences of non-negative real numbers of the same length, then we denote by $x^{y}$ the value

$$
\prod_{i=1}^{k} x_{i}^{y_{i}}
$$

where $0^{0}=1$.

We abuse the notation sometimes by using a set $T \subset \mathbb{Z}$ to denote the sequence of its elements in increasing order indexed by $\{1,2, \ldots,|T|\}$.

For instance, if $T=\{1,3,6,7\}$, we have $T_{\{3\}}=6$.

We need a straightforward technical result, which can be proved by induction in $k$.

Lemma 2.7. If $x=\left(x_{1}, x_{2}, \ldots, x_{k}\right)$ and $y=\left(y_{1}, y_{2}, \ldots, y_{k}\right)$ are two non-decreasing sequences of nonnegative real numbers of the same length and $z=\left(z_{1}, z_{2}, \ldots, z_{k}\right)$ is a permutation of the sequence $y$, then we have

$$
x^{z} \leq x^{y} .
$$

Let us now fix some notation that will be used along the proof of Theorem 1.4 and some auxiliary lemmas. This proof is based on proofs by Hästö (2002/03, Theorem 3.3) (see Theorem 4.1) and Warren (2004, Theorem 3.8).

Let $\sigma=\left(\widehat{a}, \ell_{1}, \ldots, \ell_{k}\right)$ a layered permutation with $2 \leq a \leq \ell_{1} \leq \cdots \leq \ell_{k}$, let $\ell=a+\sum_{i=1}^{k} \ell_{i}$ be the length of $\sigma$ and let $\ell_{i}=1$ for every $i \leq 0$.

For every $N \in \mathbb{N}^{*}$, define the polynomial $p_{N} \in \mathbb{R}\left[y, x_{1}, x_{2}, \ldots, x_{N}\right]$ by letting

$$
\begin{aligned}
p_{N}\left(y, x_{1}, x_{2}, \ldots, x_{N}\right) & =\frac{\prod_{i=1}^{k} \ell_{i} !}{\ell !} g_{N, \sigma}\left(y, x_{1}, 0, x_{2}, 0, x_{3}, \ldots, 0, x_{N}\right) \\
& =\sum_{u=0}^{a} \frac{y^{u}}{u !} \sum_{\left\{i_{1}, i_{2}, \ldots, i_{a-u+k}\right\}<\subset[N]}\left(\prod_{j=1}^{a-u+k} x_{i_{j}}^{\ell_{j-a+u}}\right) \\
& =\sum_{u=0}^{a} \frac{y^{u}}{u !} \sum_{I \in\left(\begin{array}{c}
{[N]} \\
a-u+k
\end{array}\right)} x_{I}^{\ell_{I-a+u+1 . k]}},
\end{aligned}
$$

where $[-a+u+1 . . k]$ denotes the set $\{-a+u+1,-a+u+2, \ldots, k\}$.

Note that

$$
\begin{gathered}
\mathfrak{L}_{N,[N] \backslash\{1\}, \sigma}=\max \left\{\frac{\ell !}{\prod_{i=1}^{k} \ell_{i} !} p_{N}\left(y, x_{1}, \ldots, x_{N}\right): y+\sum_{j=1}^{N} x_{j}=1\right. \\
\text { and } \left.\forall j \in[N], x_{j} \geq 0 \text { and } y \geq 0\right\} .
\end{gathered}
$$

Furthermore, by Theorem 2.5, we have

$$
p(\sigma)=\lim _{N \rightarrow \infty} \mathfrak{L}_{N,[N] \backslash\{1\}, \sigma}
$$


Our objective is to prove that $\mathfrak{L}_{N,[N] \backslash\{1\}, \sigma} \leq \mathfrak{L}_{N-1,[N-1] \backslash\{1\}, \sigma}$ whenever $N>k$.

To do this, we first show that we can find an optimal point $(y, x)$ for $p_{N}$ that has some "good" properties. Lemma 2.8 below shows that we that there is an optimal $(y, x)$ such that the coordinates of $x$ are increasing. To show this, we prove that if $x$ does not have increasing coordinnates and we switch the position of two consecutive decreasing coordinates, then the value of $p_{N}$ does not decrease.

Lemma 2.8. For every $N \geq k$ and $i_{0} \in[N-1]$, we have

$$
p_{N}\left(y, x_{1}, x_{2}, \ldots, x_{N}\right) \leq p_{N}\left(y, x_{1}, x_{2}, \ldots, x_{i_{0}-1}, x_{i_{0}+1}, x_{i_{0}}, x_{i_{0}+2}, \ldots, x_{N}\right),
$$

whenever $y, x_{1}, x_{2}, \ldots, x_{N} \geq 0$ and $x_{i_{0}} \geq x_{i_{0}+1}$.

Proof: Throughout this proof, we denote by $p_{N}\left(y, x_{i_{0}} \leftrightarrow x_{i_{0}+1}\right)$ the value

$$
p_{N}\left(y, x_{1}, x_{2}, \ldots, x_{i_{0}-1}, x_{i_{0}+1}, x_{i_{0}}, x_{i_{0}+2}, \ldots, x_{N}\right)
$$

where we exchange the variables $x_{i_{0}}$ and $x_{i_{0}+1}$ of $p_{N}$.

We now study the difference $p_{N}\left(y, x_{i_{0}} \leftrightarrow x_{i_{0}+1}\right)-p_{N}\left(y, x_{1}, \ldots, x_{N}\right)$.

Note that the summands in which $I$ does not contain either $i_{0}$ or $i_{0}+1$ cancel out.

Furthermore, the summands of $p_{N}\left(y, x_{i_{0}} \leftrightarrow x_{i_{0}+1}\right)$ in which $I$ contains $i_{0}$ but does not contain $i_{0}+1$ cancel out with the summands of $p_{N}\left(y, x_{1}, \ldots, x_{N}\right)$ in which $I$ contains $i_{0}+1$ but does not contain $i_{0}$ (because in the first, the values $x_{i_{0}}$ and $x_{i_{0}+1}$ are swapped).

Analogously, the summands of $p_{N}\left(y, x_{i_{0}} \leftrightarrow x_{i_{0}+1}\right)$ in which $I$ contains $i_{0}+1$ but does not contain $i_{0}$ cancel out with the summands of $p_{N}\left(y, x_{1}, \ldots, x_{N}\right)$ in which $I$ contains $i_{0}$ but does not contain $i_{0}+1$.

This means that we have

$$
\begin{aligned}
& p_{N}\left(y, x_{i_{0}} \leftrightarrow x_{i_{0}+1}\right)-p_{N}\left(y, x_{1}, x_{2}, \ldots, x_{N}\right)
\end{aligned}
$$

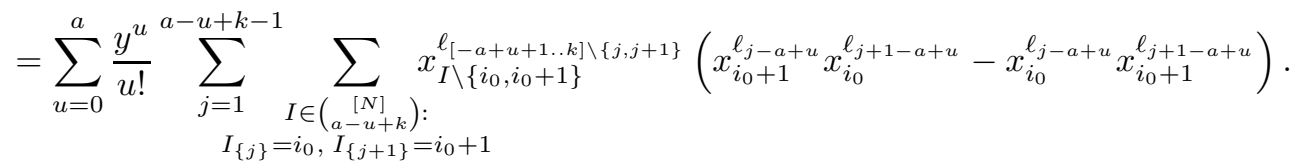

Now, since $x_{i_{0}} \geq x_{i_{0}+1} \geq 0$ and

$$
\ell_{k} \geq \ell_{k-1} \geq \cdots \geq \ell_{1} \geq \ell_{0} \geq \ell_{-1} \geq \cdots \geq \ell_{1-a}
$$

we have $x_{i_{0}+1}^{\ell_{t}} x_{i_{0}}^{\ell_{t+1}} \geq x_{i_{0}}^{\ell_{t}} x_{i_{0}+1}^{\ell_{t+1}}$ for every $t<k$, hence

$$
p_{N}\left(y, x_{i_{0}} \leftrightarrow x_{i_{0}+1}\right)-p_{N}\left(y, x_{1}, x_{2}, \ldots, x_{N}\right) \geq 0
$$

Lemma 2.8 immediately implies that we may add the restriction

$$
x_{1} \leq x_{2} \leq \cdots \leq x_{N}
$$

to the maximum that defines $\mathfrak{L}_{N,[N] \backslash\{1\}, \sigma}$.

Lemma 2.9 below, shows that there is an optimal point $(y, x)$ such that $y \leq x_{N-k+1}$. To do this, we use a calculus argument to show that if $y>x_{N-k+1}$, then there exists $\epsilon>0$ such that if we increase $y$ by $\epsilon$ and decrease $x_{N-k+1}$ by $\epsilon$, then the value of $p_{N}$ increases. 
Lemma 2.9. For every $N>k$, we may also add the restriction $y \leq x_{N-k+1}$ to the maximum that defines $\mathfrak{L}_{N,[N] \backslash\{1\}, \sigma}$, i.e., we have

$$
\begin{gathered}
\mathfrak{L}_{N,[N] \backslash\{1\}, \sigma}=\max \left\{\frac{\ell !}{\prod_{i=1}^{k} \ell_{i} !} p_{N}\left(y, x_{1}, x_{2}, \ldots, x_{N}\right): 0 \leq x_{1} \leq x_{2} \leq \cdots \leq x_{N}\right. \text { and } \\
\left.0 \leq y \leq x_{N-k+1} \text { and } y+\sum_{j=1}^{N} x_{j}=1\right\}
\end{gathered}
$$

Proof: Suppose not and let $y, x_{1}, x_{2}, \ldots, x_{N} \geq 0$ be such that $x_{1} \leq x_{2} \leq \cdots \leq x_{N} ; y+\sum_{j=1}^{N} x_{j}=1$ and

$$
\mathfrak{L}_{N,[N] \backslash\{1\}, \sigma}=\frac{\ell !}{\prod_{i=1}^{k} \ell_{i} !} p_{N}\left(y, x_{1}, x_{2}, \ldots, x_{N}\right),
$$

and suppose that $y>x_{N-k+1}$.

For every $t \in \mathbb{R}$, let

$$
f(t)=p_{N}\left(y-t, x_{1}, x_{2}, \ldots, x_{N-k}, x_{N-k+1}+t, x_{N-k+2}, \ldots, x_{N}\right),
$$

and note that, for every $0 \leq t \leq y$, we have

$$
\mathfrak{L}_{N,[N] \backslash\{1\}, \sigma} \geq \frac{\ell !}{\prod_{i=1}^{k} \ell_{i} !} f(t),
$$

with equality if $t=0$ (we may lose the condition $x_{1} \leq x_{2} \leq \cdots \leq x_{N}$ for $t>0$ ).

Since $f$ is differentiable, we must have $f^{\prime}(0) \leq 0$. But note that

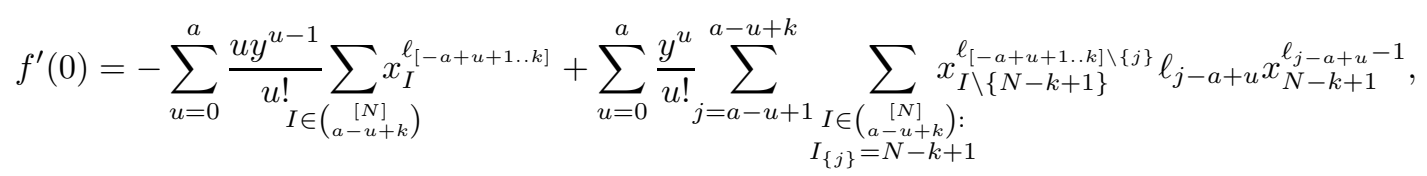

where the first sum groups terms that derived from $y-t$ and the second sum groups terms derived from $x_{N-k+1}+t$.

We now split the first sum according to summands in which $I$ has or not the element $N-k+1$, obtaining

$$
\begin{aligned}
& \sum_{u=0}^{a} \frac{u y^{u-1}}{u !} \sum_{I \in\left(\begin{array}{c}
{[N]} \\
a-u+k
\end{array}\right)} x_{I-a+u+1 \ldots k]}^{\ell^{[-a+k}}
\end{aligned}
$$

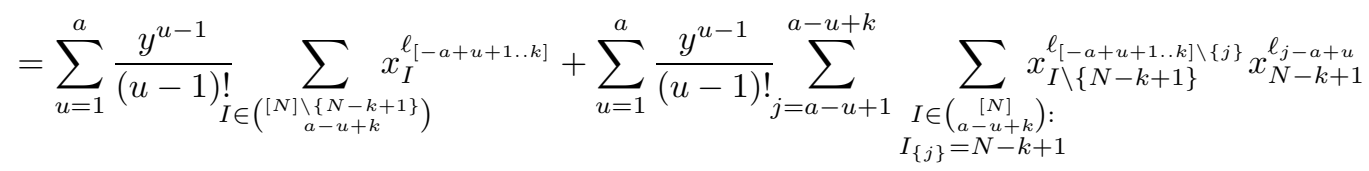

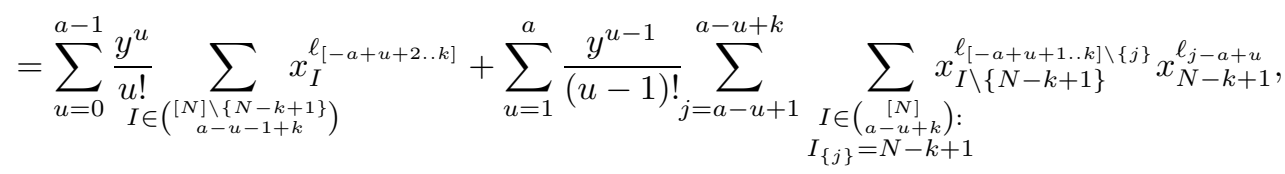


where in the last equality we applied the change of variables $u \rightarrow u+1$ to the first sum.

Grouping back in the original equation yields

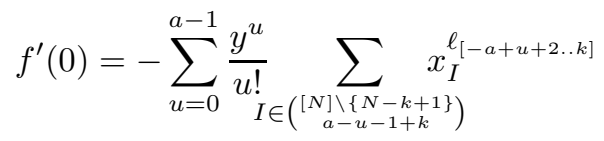

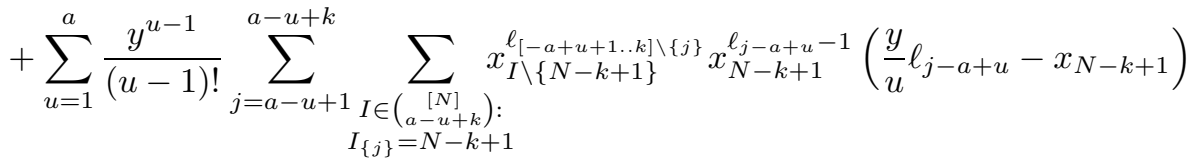

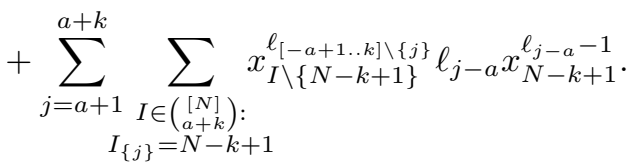

Now we give a lower bound for the last two sums. First, note that, for every $u \in[a]$ and every $a-u+1 \leq$ $j \leq a-u+k$, we have

$$
\frac{y}{u} \ell_{j-a+u}-x_{N-k+1} \geq \frac{y}{u} \ell_{1}-x_{N-k+1} \geq 0,
$$

since $u \leq a \leq \ell_{1}$ and $y \geq x_{N-k+1}$.

Using the first inequality and Lemma 2.7 , we have

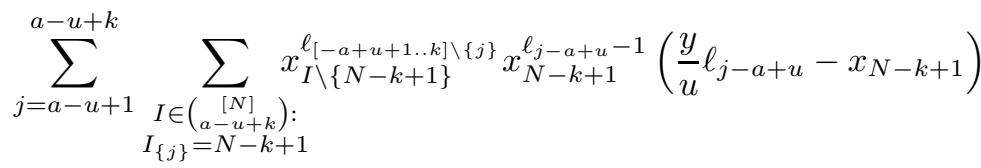

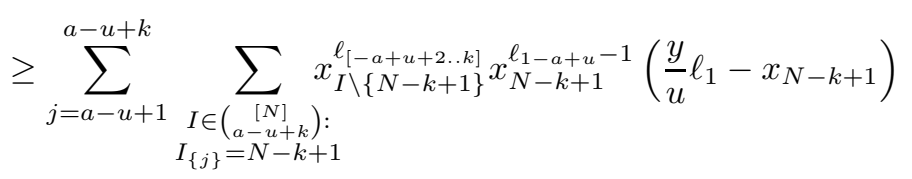

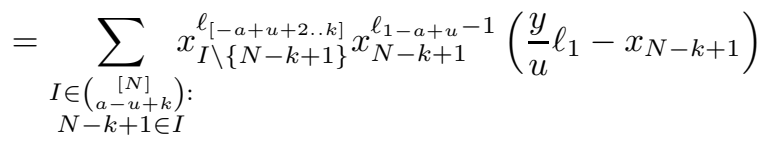

$$
\begin{aligned}
& =\sum_{I \in\left(\begin{array}{c}
{[N] \backslash\{N-k+1\}} \\
a-u-1+k
\end{array}\right)} x_{I}^{\ell_{[-a+u+2 . k]}} x_{N-k+1}^{\ell_{1-a+u}-1}\left(\frac{y}{u} \ell_{1}-x_{N-k+1}\right),
\end{aligned}
$$

where in the last equality we applied the change of variables $I \rightarrow I \backslash\{N-k+1\}$. 
Analogously, we have

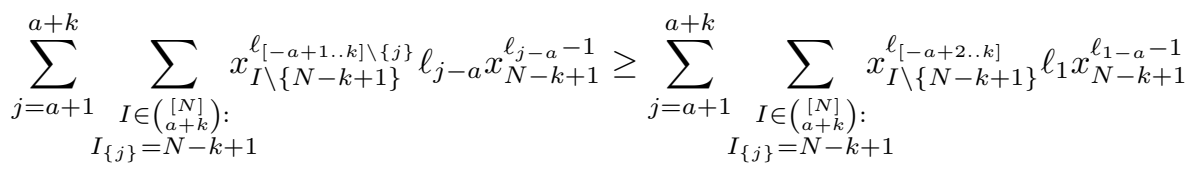

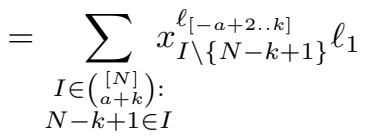

$$
\begin{aligned}
& =\sum_{I \in\left(\begin{array}{c}
{[N] \backslash\{N-k+1\}} \\
a-1+k
\end{array}\right)} x_{I}^{\ell_{[-a+2 . . k]}} \ell_{1},
\end{aligned}
$$

since $\ell_{1-a}=1$.

Gathering all up, we have

$$
\begin{aligned}
& f^{\prime}(0) \geq-\sum_{u=0}^{a-1} \frac{y^{u}}{u !} \sum_{I \in\left(\begin{array}{c}
[N] \backslash N-k+1\} \\
a-u-1+k
\end{array}\right)} x_{I-a+u+2 \ldots k]}^{\ell[-1+k} \\
& +\sum_{u=1}^{a} \frac{y^{u-1}}{(u-1) !} \sum_{I \in\left(\begin{array}{c}
{[N] \backslash\{N-k+1\}} \\
a-u-1+k
\end{array}\right)} x_{I}^{\ell_{[-a+u+2 . . k]}} x_{N-k+1}^{\ell_{1}-a+u-1}\left(\frac{y}{u} \ell_{1}-x_{N-k+1}\right) \\
& +\sum_{I \in\left(\begin{array}{c}
{[N] \backslash\{N-k+1\}} \\
a-1+k
\end{array}\right)} x_{I}^{\ell^{[-a+2 . . k]} \ell_{1}} \\
& =\sum_{u=1}^{a-1} \frac{y^{u-1}}{(u-1) !} \sum_{\substack{I \in\left(\begin{array}{c}
{[N] \backslash\{N-k+1\} \\
a-u-1+k}
\end{array}\right) \\
u}}\left(\frac{y}{u} \ell_{1}-x_{N-k+1}-\frac{y}{u}\right) x_{I}^{\ell_{I-a+u+2 . . k]}} \\
& +\frac{y^{a-1}}{(a-1) !} \sum_{I \in\left(\begin{array}{c}
{[N] \backslash\{N-k+1\}} \\
k-1
\end{array}\right)}\left(\frac{y}{a} \ell_{1}-x_{N-k+1}\right) x_{I}^{\ell_{I 2 . k]}} x_{N-k+1}^{\ell_{1}-1} \\
& +\sum_{I \in\left(\begin{array}{c}
{[N] \backslash\{N-k+1\}} \\
a-1+k
\end{array}\right)} x_{I}^{\ell[-a+2 \ldots k]}\left(\ell_{1}-1\right) .
\end{aligned}
$$

Note that, for every $u \in[a-1]$, we have

$$
\frac{y}{u} \ell_{1}-x_{N-k+1}-\frac{y}{u}=\frac{y}{u}\left(\ell_{1}-1\right)-x_{N-k+1} \geq \frac{\ell_{1}-1}{a-1} y-x_{N-k+1} \geq y-x_{N-k+1}>0,
$$

since $\ell_{1} \geq a$.

Furthermore, note that

$$
\frac{y}{a} \ell_{1}-x_{N-k+1} \geq y-x_{N-k+1}>0,
$$

and also $\ell_{1}-1>0$, hence we have $f^{\prime}(0)>0$, which is a contradiction. 
Lemma 2.10 below shows that it is enough to consider the Extended Price Bound of order $k$ relative to $[k] \backslash\{1\}$. To do this, we start with an optimal point $(y, x)$ as provided by Lemma 2.9 and we show that if we join the smaller layer (corresponding to $x_{1}$ ) with the antilayer (corresponding to $y$ ) then the objective value does not decrease, i.e., we have $\mathfrak{L}_{N,[N] \backslash\{1\}, \sigma} \leq \mathfrak{L}_{N-1,[N-1] \backslash\{1\}, \sigma}$.

Lemma 2.10. For every $N>k$, we have

$$
\mathfrak{L}_{N,[N] \backslash\{1\}, \sigma} \leq \mathfrak{L}_{N-1,[N-1] \backslash\{1\}, \sigma}
$$

Proof of Lemma 2.10: From Lemma 2.9, we know that there exist $y, x_{1}, x_{2}, \ldots, x_{N} \geq 0$ such that $x_{1} \leq$ $x_{2} \leq \cdots \leq x_{N}, y+\sum_{j=1}^{N} x_{j}=1, y \leq x_{N-k+1}$, and

$$
\mathfrak{L}_{N,[N] \backslash\{1\}, \sigma}=\frac{\ell !}{\prod_{i=1}^{k} \ell_{i} !} p_{N}\left(y, x_{1}, x_{2}, \ldots, x_{N}\right) .
$$

We now consider what happens when we use the Extended Price Polynomial of order $N-1$ on the point $\left(y+x_{1}, x_{2}, x_{3}, \ldots, x_{N}\right)$. This corresponds to merging the first two blocks of $p_{N}$ into an antilayer.

Note that

$$
\mathfrak{L}_{N-1,[N-1] \backslash\{1\}, \sigma} \geq \frac{\ell !}{\prod_{i=1}^{k} \ell_{i} !} p_{N-1}\left(y+x_{1}, x_{2}, x_{3}, \ldots, x_{N}\right),
$$

hence it is enough to prove that

$$
p_{N-1}\left(y+x_{1}, x_{2}, x_{3}, \ldots, x_{N}\right) \geq p_{N}\left(y, x_{1}, x_{2}, \ldots, x_{N}\right) .
$$

To do this, we reorganize the summands that occur in $p_{N-1}$ and in $p_{N}$ in a way that, when we compute their difference, each summand of $p_{N-1}$ corresponds to a smaller summand of $p_{N}$.

First, we split the sum in the definition of $p_{N}$ according to summands that have $x_{1}$ and summands that do not. So note that

$$
\begin{aligned}
& p_{N}\left(y, x_{1}, x_{2}, \ldots, x_{N}\right) \\
& =\sum_{u=0}^{a} \frac{y^{u}}{u !} \sum_{\substack{I \in] \\
I \in-u+k}} x_{I}^{\ell_{[-a+u+1 . . k]}}
\end{aligned}
$$

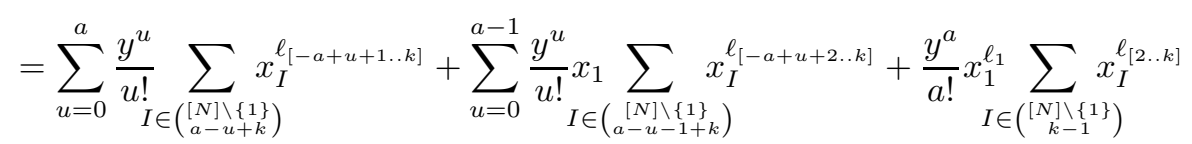

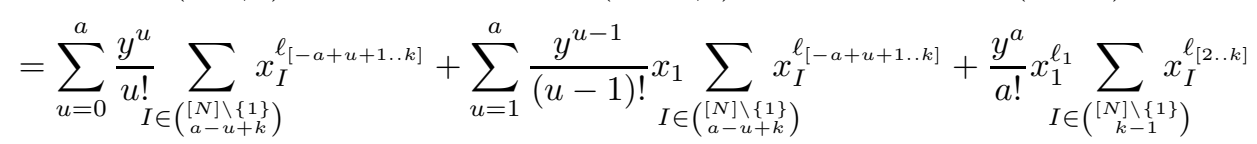

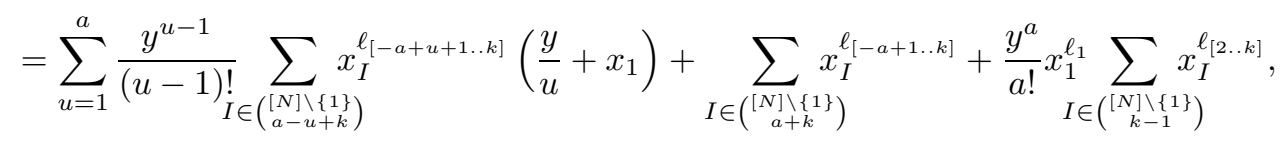


where in the third equality we applied the change of variables $u \rightarrow u-1$ to the second sum.

On the other hand, we can also separate the summand of $p_{N-1}$ that has $u=0$, obtaining

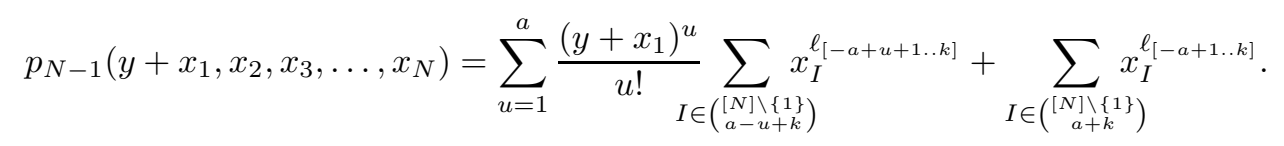

So, computing the difference, we have

$$
\begin{aligned}
& p_{N-1}\left(y+x_{1}, x_{2}, x_{3}, \ldots, x_{N}\right)-p_{N}\left(y, x_{1}, x_{2}, \ldots, x_{N}\right)
\end{aligned}
$$

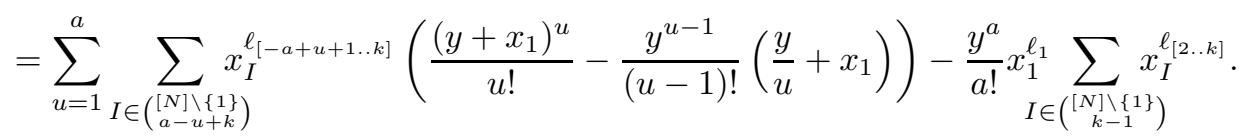

Now, for every $u \in[a]$, we have

$$
\left(\frac{\left(y+x_{1}\right)^{u}}{u !}-\frac{y^{u-1}}{(u-1) !}\left(\frac{y}{u}+x_{1}\right)\right)=\frac{1}{u !}\left(\left(y+x_{1}\right)^{u}-y^{u}-u x_{1} y^{u-1}\right)=\frac{1}{u !} \sum_{v=0}^{u-2}\left(\begin{array}{l}
u \\
v
\end{array}\right) y^{v} x_{1}^{u-v} \geq 0 .
$$

This yields

$$
\begin{aligned}
& p_{N-1}\left(y+x_{1}, x_{2}, x_{3}, \ldots, x_{N}\right)-p_{N}\left(y, x_{1}, x_{2}, \ldots, x_{N}\right) \\
\geq & \frac{1}{a !} \sum_{v=0}^{a-2}\left(\begin{array}{l}
a \\
v
\end{array}\right) y^{v} x_{1}^{a-v} \sum_{I \in\left(\begin{array}{c}
{[N] \backslash\{1\}} \\
k
\end{array}\right)} x_{I}^{\ell_{[k]}}-\frac{y^{a}}{a !} x_{1}^{\ell_{1}} \sum_{I \in\left(\begin{array}{c}
{[N] \backslash\{1\}} \\
k-1
\end{array}\right)} x_{I}^{\ell_{[2 . . k]}} .
\end{aligned}
$$

Note now that

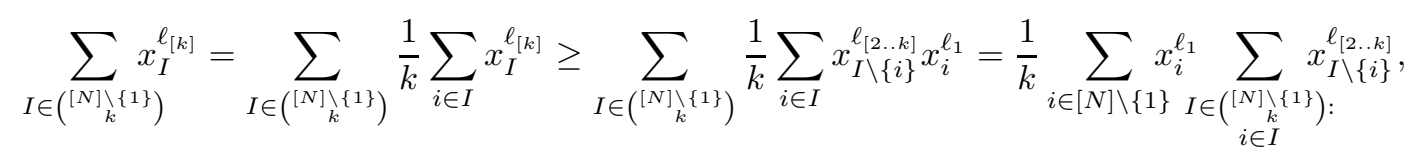

where the inequality follows from Lemma 2.7.

Now we can apply the change of variables $I \rightarrow I \backslash\{i\}$ and obtain

$$
\begin{aligned}
& \sum_{I \in\left(\begin{array}{l}
{[N] \backslash\{1\}} \\
k
\end{array}\right)} x_{I}^{\ell_{[k]}} \geq \frac{1}{k} \sum_{i \in[N] \backslash\{1\}} x_{i}^{\ell_{1}} \sum_{\substack{[\in([N] \backslash\{1\} \\
k \neq 1 \\
i \notin I}} x_{I}^{\ell_{[}[2 . . k]}
\end{aligned}
$$

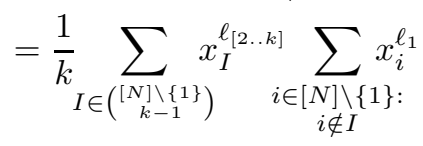

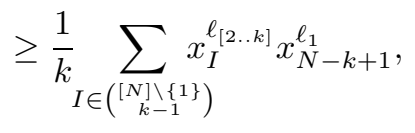


where the last inequality follows from the fact that the inner sum has to contain at least one of the summands $x_{N-k+1}^{\ell_{1}}, x_{N-k+2}^{\ell_{1}}, \ldots, x_{N}^{\ell_{1}}$ and they are all greater or equal to $x_{N-k+1}^{\ell_{1}}$.

Using this new inequality, we have

$$
\begin{gathered}
p_{N-1}\left(y+x_{1}, x_{2}, x_{3}, \ldots, x_{N}\right)-p_{N}\left(y, x_{1}, x_{2}, \ldots, x_{N}\right) \\
\geq \frac{1}{a !}\left(\frac{1}{k} \sum_{v=0}^{a-2}\left(\begin{array}{l}
a \\
v
\end{array}\right) y^{v} x_{1}^{a-v} x_{N-k+1}^{\ell_{1}}-y^{a} x_{1}^{\ell_{1}}\right) \sum_{\substack{[N] \backslash\{1\} \\
k-1}} x_{I}^{\ell_{[2 . k]}} .
\end{gathered}
$$

Finally, since $x_{N-k+1} \geq x_{1}$ and $x_{N-k+1} \geq y$, we have

$$
\begin{aligned}
\frac{1}{k} \sum_{u=0}^{a-2}\left(\begin{array}{l}
a \\
u
\end{array}\right) y^{u} x_{1}^{a-u} x_{N-k+1}^{\ell_{1}}-y^{a} x_{1}^{\ell_{1}} & \geq \frac{1}{k} \sum_{u=0}^{a-2}\left(\begin{array}{l}
a \\
u
\end{array}\right) y^{a} x_{1}^{\ell_{1}}-y^{a} x_{1}^{\ell_{1}} \\
& =\frac{2^{a}-a-1}{k} y^{a} x_{1}^{\ell_{1}}-y^{a} x_{1}^{\ell_{1}} \\
& \geq 0,
\end{aligned}
$$

where the last inequality follows from the hypothesis that $2^{a}-a-1 \geq k$.

Therefore we have

$$
p_{N-1}\left(y+x_{1}, x_{2}, x_{3}, \ldots, x_{N}\right) \geq p_{N}\left(y, x_{1}, x_{2}, \ldots, x_{N}\right) .
$$

Finally, Theorem 1.4 follows by a simple argument.

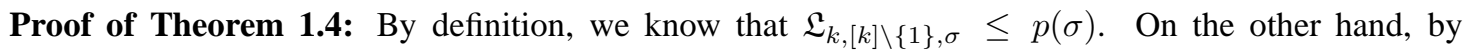
Lemma 2.10 we have

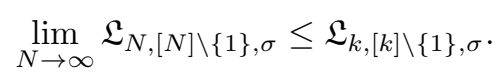

Then, by Theorem 2.5, we conclude that $p(\sigma)=\mathfrak{L}_{k,[k] \backslash\{1\}, \sigma}$.

The value

$$
p(\sigma)=\frac{|\sigma| !}{|\sigma|^{|\sigma|}} \frac{a^{a}}{a !} \prod_{i=1}^{k} \frac{\ell_{i}^{\ell_{i}}}{\ell_{i} !} .
$$

can now be computed with a standard calculus argument involving Lagrange multipliers.

The corollary below follows from a simple argument presented by Hästö (2002/03, Lemma 3.4) (see Corollary 4.2). In simple words, the corollary below says that Theorem 1.4 remains valid even if we change the order of the blocks.

Corollary 2.11. Let $a, k \in \mathbb{N}^{*}$ be positive integers such that $a \geq 2$ and $2^{a}-a-1 \geq k$.

Let also $\sigma$ be a layered permutation having exactly one antilayer of length $a$ and $k$ layers of lengths at least two. Furthermore, suppose the lengths of all these $k$ layers are greater or equal to $a$.

If the antilayer is the $j$-th block of $\sigma$ and $j \leq k$, then we have

$$
p(\sigma)=\mathfrak{L}_{k,[k] \backslash\{j\}, \sigma} .
$$


Proof: Let $\ell_{1} \leq \ell_{2} \leq \cdots \leq \ell_{k}$ be the lengths of the layers of $\sigma$ of length at least 2 and let $\sigma^{\prime}$ be the permutation of block sequence $\left(\widehat{a}, \ell_{1}, \ell_{2}, \ldots, \ell_{k}\right)$.

First we prove that for every $N \in \mathbb{N}^{*}$, we have $\mathfrak{L}_{N, \sigma} \leq \mathfrak{L}_{N, \sigma^{\prime}}$ (note that we are using the common Price Bound).

Let $\left(r_{1}, r_{2}, \ldots, r_{m}\right)$ be the layer sequence of $\sigma$ (note that the sequence includes the $\ell_{i}$ 's in some order and includes a sequence of 1's of length $a$, hence $m=k+a$ ).

Let $x=\left(x_{1}, x_{2}, \ldots, x_{N}\right)$ be such that $x_{i} \geq 0$ for every $i \in[N], \sum_{i=1}^{N} x_{i}=1$, and

$$
q_{N, \sigma}(x)=\mathfrak{L}_{N, \sigma} .
$$

Finally, for every sequence of real numbers $s$, let $s_{\leq}$be the ordering of $s$ (i.e., the sequence $s_{\leq}$is non-decreasing and has the same elements of $s$ ).

Note now that

$$
\begin{aligned}
q_{N, \sigma}(x) & =\frac{|\sigma| !}{\prod_{i=1}^{m} r_{i} !} \sum_{I \in\left(\begin{array}{c}
{[N]} \\
m
\end{array}\right)} x_{I}^{r_{[m]}} \leq \frac{|\sigma| !}{\prod_{i=1}^{m} r_{i} !} \sum_{I \in\left(\begin{array}{c}
{[N]} \\
m
\end{array}\right)}\left(x_{I}\right)_{\leq}^{\left(r_{[m]}\right)^{[}} \leq \\
& =\frac{|\sigma| !}{\prod_{i=1}^{m} r_{i} !} \sum_{I \in\left(\begin{array}{c}
{[N]} \\
m
\end{array}\right)}\left(x_{\leq}\right)_{I}^{\left(r_{[m]}\right)} \leq=q_{N, \sigma^{\prime}}\left(x_{\leq}\right) \leq \mathfrak{L}_{N, \sigma^{\prime}} .
\end{aligned}
$$

Therefore, we have $p(\sigma) \leq p\left(\sigma^{\prime}\right)$.

Now, from Theorem 1.4, we know that $p\left(\sigma^{\prime}\right)=\mathfrak{L}_{k,[k] \backslash\{1\}, \sigma^{\prime}}$, so let $y, x_{1}, x_{2}, \ldots, x_{k} \geq 0$ be such that $y+\sum_{i=1}^{k} x_{i}=1$ and

$$
g_{k, \sigma^{\prime}}\left(y, x_{1}, 0, x_{2}, 0, x_{3}, \ldots, 0, x_{k}\right)=\mathfrak{L}_{k,[k] \backslash\{1\}, \sigma^{\prime}} .
$$

Let $\tau$ be a permutation such that the block sequence of $\sigma$ is

$$
\left(\ell_{\tau(1)}, \ell_{\tau(2)}, \ldots, \ell_{\tau(j-1)}, \widehat{a}, \ell_{\tau(j)}, \ldots, \ell_{\tau(k)}\right) .
$$

Note that

$$
\begin{aligned}
p\left(\sigma^{\prime}\right) & =\mathfrak{L}_{k,[k] \backslash\{1\}, \sigma^{\prime}}=g_{k, \sigma^{\prime}}\left(y, x_{1}, 0, x_{2}, 0, x_{3}, \ldots, 0, x_{k}\right) \\
& =\frac{\left|\sigma^{\prime}\right| !}{a ! \prod_{i=1}^{k} \ell_{i} !} y^{a} x^{\ell_{[k]}}=\frac{|\sigma| !}{a ! \prod_{i=1}^{k} \ell_{i} !} y^{a} \prod_{i=1}^{k} x_{\tau(i)}^{\ell_{\tau(i)}} \\
& =g_{k, \sigma}\left(0, x_{\tau(1)}, 0, x_{\tau(2)}, \ldots, 0, x_{\tau(j-1)}, y, x_{\tau(j)}, 0, x_{\tau(j+1)}, \ldots, 0, x_{\tau(k)}\right) \\
& \leq \mathfrak{L}_{k,[k] \backslash\{j\}, \sigma} \leq p(\sigma) .
\end{aligned}
$$

Therefore $p(\sigma)=p\left(\sigma^{\prime}\right)=\mathfrak{L}_{k,[k] \backslash\{j\}, \sigma}$.

Remark. Note that we do not allow the antilayer to be the last block simply because the Extended Price Polynomial does not end with an antilayer.

If the antilayer happens to be the last block of $\sigma$, we cannot apply Corollary 2.11 directly, but it is easy to see that $\sigma$ must have the same packing density as the permutation whose block sequence is the reverse of the block sequence of $\sigma$ (and this permutation does not have an antilayer as its last block). 


\section{Minimization problem}

We now study the dual problem of minimizing the density of permutations asymptotically.

Definition 3.1. The Minimization Price Bound of order $n \in \mathbb{N}^{*}$ for a linear combination of layered permutations $f$ is the value

$$
\mathfrak{U}_{n, f}=\min \left\{q_{n, f}\left(x_{1}, x_{2}, \ldots, x_{n}\right): \sum_{j=1}^{n} x_{j}=1 \text { and } \forall j \in[n], x_{j} \geq 0\right\} .
$$

Remark. This minimum exists by a compactness argument.

For the Minimization Price Bound, we have the following analogous results.

Proposition 3.2. If $f \in \mathbb{R} \mathfrak{S}$ is a conical combination of layered permutations, then, for every $n \in \mathbb{N}^{*}$, we have $\mathfrak{U}_{n, f} \geq \mathfrak{U}_{n+1, f}$ and

$$
\lim _{n \rightarrow \infty} \mathfrak{U}_{n, f} \geq p^{\prime \prime}(f)
$$

Proof : Analogous to the proof of Proposition 2.1.

Theorem 3.3. If $f \in \mathbb{R} \mathfrak{S}$ is a conical combination of layered permutations, then we have

$$
\lim _{n \rightarrow \infty} \mathfrak{U}_{n, f}=p^{\prime \prime}(f) .
$$

Proof : Analogous to the proof of Corollary 2.2.

Remark. An analogous result is also valid for Extended Price Polynomials if we define the Minimization Extended Price Bound.

Proof of Theorem 1.9: First note that, for every $N \in \mathbb{N}^{*}$, we have

$$
q_{N, \operatorname{Id}_{l}+\operatorname{Rev}_{k}}\left(x_{1}, x_{2}, \ldots, x_{N}\right)=\ell ! \sum_{I \in\left(\begin{array}{c}
{[N]} \\
\ell
\end{array}\right)} \prod_{i \in I} x_{i}+\sum_{i \in[N]} x_{i}^{k}
$$

Let $x_{1}, x_{2}, \ldots, x_{N} \geq 0$ with $\sum_{i=1}^{N} x_{i}=1$ be such that

$$
q_{N, \operatorname{Id}_{\ell}+\operatorname{Rev}_{k}}\left(x_{1}, x_{2}, \ldots, x_{N}\right)=\mathfrak{U}_{N, \mathrm{Id}_{\ell}+\operatorname{Rev}_{k}},
$$

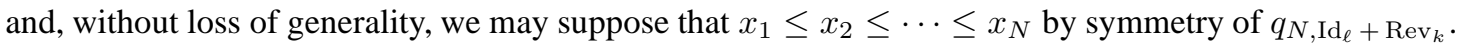

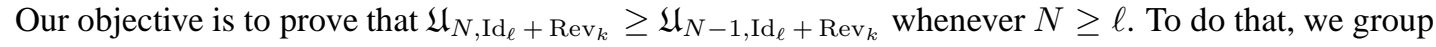
the summands in $q_{N, \mathrm{Id}_{\ell}}+\operatorname{Rev}_{k}$ and $q_{N-1, \mathrm{Id}_{\ell}}+\operatorname{Rev}_{k}$ according to which of $x_{1}$ and/or $x_{2}$ they contain. So note that

$$
\begin{aligned}
& q_{N, \mathrm{Id}_{\ell}+\operatorname{Rev}_{k}}\left(x_{1}, x_{2}, \ldots, x_{N}\right)
\end{aligned}
$$

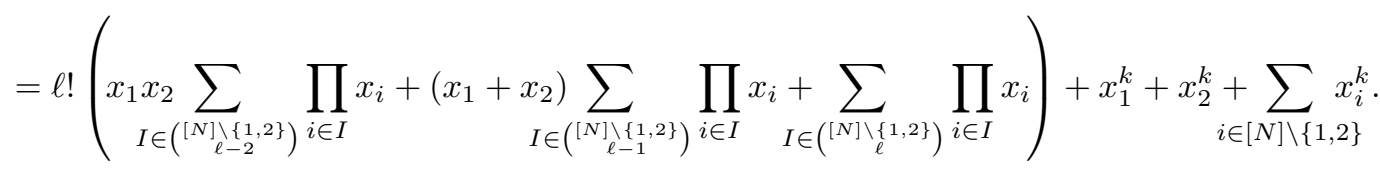


On the other hand, we have

$$
\begin{aligned}
& q_{N-1, \mathrm{Id}_{\ell}+\operatorname{Rev}_{k}}\left(x_{1}+x_{2}, x_{3}, x_{4}, \ldots, x_{N}\right) \\
& \left.=\ell !\left(x_{1}+x_{2}\right) \sum_{I \in\left({ }^{[N] \backslash\{1,2\}}\right)} \prod_{\substack{i \in I \\
\ell-1}} x_{i}+\sum_{I \in\left({ }^{[N] \backslash\{1,2\}}\right)} \prod_{\substack{i \in I \\
\ell}} x_{i}\right)+\left(x_{1}+x_{2}\right)^{k}+\sum_{i \in[N] \backslash\{1,2\}} x_{i}^{k} .
\end{aligned}
$$

Subtracting the polynomials then yields

$$
\begin{aligned}
& q_{N, \mathrm{Id}_{\ell}}+\operatorname{Rev}_{k}\left(x_{1}, x_{2}, \ldots, x_{N}\right)-q_{N-1, \mathrm{Id}_{\ell}+\operatorname{Rev}_{k}}\left(x_{1}+x_{2}, x_{3}, x_{4}, \ldots, x_{N}\right) \\
= & \ell ! x_{1} x_{2} \sum_{I \in([N] \backslash\{1,2\}} \prod_{i \in I} x_{i}+x_{1}^{k}+x_{2}^{k}-\left(x_{1}+x_{2}\right)^{k} \\
\geq & \ell ! x_{1} x_{2} \sum_{I \in\left({ }^{[N] \backslash\{1,2\}}\right)} x_{2}^{\ell-2}+x_{1}^{k}+x_{2}^{k}-\left(x_{1}+x_{2}\right)^{k} \\
= & \ell ! x_{1} x_{2}^{\ell-1}\left(\begin{array}{c}
N-2 \\
\ell-2
\end{array}\right)-\sum_{v=1}^{k-1}\left(\begin{array}{l}
k \\
v
\end{array}\right) x_{1}^{v} x_{2}^{k-v} \\
\geq & \ell ! x_{1} x_{2}^{\ell-1}\left(\begin{array}{c}
N-2 \\
\ell-2
\end{array}\right)-\sum_{v=1}^{k-1}\left(\begin{array}{c}
k \\
v
\end{array}\right) x_{1} x_{2}^{k-1} \\
= & x_{1} x_{2}^{\ell-1}\left(\ell !\left(\begin{array}{c}
N-2 \\
\ell-2
\end{array}\right)-\left(2^{k}-2\right) x_{2}^{k-\ell}\right) .
\end{aligned}
$$

Now, since $x_{2}$ is the second smallest of the $x_{i}$ 's, we have $x_{2} \leq 2 / N$, hence, since $k \geq \ell$, we have

$$
\begin{aligned}
& q_{N, \operatorname{Id}_{\ell}}+\operatorname{Rev}_{k}\left(x_{1}, x_{2}, \ldots, x_{N}\right)-q_{N-1, \operatorname{Id}_{\ell}}+\operatorname{Rev}_{k}\left(x_{1}+x_{2}, x_{3}, x_{4}, \ldots, x_{N}\right) \\
& \geq x_{1} x_{2}^{\ell-1}\left(\ell !\left(\begin{array}{c}
N-2 \\
\ell-2
\end{array}\right)-\left(2^{k}-2\right)\left(\frac{2}{N}\right)^{k-\ell}\right) \text {. }
\end{aligned}
$$

To prove that this value is non-negative, we consider three cases.

Case 1. If $N \geq \ell+1$, then $N \geq 4$, hence

$$
\begin{aligned}
\ell !\left(\begin{array}{c}
N-2 \\
\ell-2
\end{array}\right)-\left(2^{k}-2\right)\left(\frac{2}{N}\right)^{k-\ell} & \geq \ell !\left(\begin{array}{c}
N-2 \\
\ell-2
\end{array}\right)-\left(2^{k}-2\right) 2^{\ell-k} \\
& \geq \ell ! \frac{(N-\ell+1)^{\ell-2}}{(\ell-2) !}-2^{\ell} \\
& =\ell(\ell-1)(N-\ell+1)^{\ell-2}-2^{\ell}
\end{aligned}
$$

and since $\ell \geq 3$ and $N-\ell+1 \geq 2$, this value is non-negative.

Case 2. If $N=\ell$ and $\ell \geq 4$, then

$$
\begin{aligned}
\ell !\left(\begin{array}{c}
N-2 \\
\ell-2
\end{array}\right)-\left(2^{k}-2\right)\left(\frac{2}{N}\right)^{k-\ell} & \geq \ell !\left(\begin{array}{c}
N-2 \\
\ell-2
\end{array}\right)-\left(2^{k}-2\right) 2^{\ell-k} \\
& =\ell !-2^{\ell}+2^{\ell-k+1}
\end{aligned}
$$


which is also non-negative.

Case 3. If $N=\ell=3$, then we have

$$
\begin{aligned}
& q_{3, \mathrm{Id}_{3}+\operatorname{Rev}_{k}}\left(x_{1}, x_{2}, x_{3}\right)-q_{2, \mathrm{Id}_{3}, \operatorname{Rev}_{k}}\left(x_{1}+x_{2}, x_{3}\right) \\
& =3 ! x_{1} x_{2} x_{3}+x_{1}^{k}+x_{2}^{k}-\left(x_{1}+x_{2}\right)^{k} \\
& =6 x_{1} x_{2} x_{3}-\sum_{v=1}^{k-1}\left(\begin{array}{l}
k \\
v
\end{array}\right) x_{1}^{v} x_{2}^{k-v} \\
& =x_{1} x_{2}\left(6 x_{3}-k x_{1}^{k-2}-k x_{2}^{k-2}-\sum_{v=2}^{k-2}\left(\begin{array}{l}
k \\
v
\end{array}\right) x_{1}^{v-1} x_{2}^{k-v-1}\right) \\
& =x_{1} x_{2}\left(6 x_{3}-k x_{1}^{k-2}-k x_{2}^{k-2}-\sum_{v=1}^{k-3}\left(\begin{array}{c}
k \\
v+1
\end{array}\right) x_{1}^{v} x_{2}^{k-v-2}\right) \\
& =x_{1} x_{2}\left(6 x_{3}-k \sum_{v=0}^{k-2}\left(\begin{array}{c}
k-2 \\
v
\end{array}\right) x_{1}^{v} x_{2}^{k-2-v}+k \sum_{v=1}^{k-3}\left(\begin{array}{c}
k-2 \\
v
\end{array}\right) x_{1}^{v} x_{2}^{k-2-v}-\sum_{v=1}^{k-3}\left(\begin{array}{c}
k \\
v+1
\end{array}\right) x_{1}^{v} x_{2}^{k-2-v}\right) \\
& =x_{1} x_{2}\left(6 x_{3}-k\left(x_{1}+x_{2}\right)^{k-2}+\sum_{v=1}^{k-3}\left(k\left(\begin{array}{c}
k-2 \\
v
\end{array}\right)-\left(\begin{array}{c}
k \\
v+1
\end{array}\right)\right) x_{1}^{v} x_{2}^{k-v}\right) \text {, }
\end{aligned}
$$

where in the fourth equality, we applied the change of variables $v \rightarrow v+1$.

Let us prove that this value is non-negative.

Since $x_{3}$ is the greatest of the $x_{i}$ 's, we have $x_{3} \geq 1 / 3$, hence $x_{1}+x_{2} \leq 2 / 3$. Since $k(2 / 3)^{k-2}$ is a non-increasing function of $k$ when $k \geq 2$, we have

$$
6 x_{3}-k\left(x_{1}+x_{2}\right)^{k-2} \geq 2-k\left(\frac{2}{3}\right)^{k-2} \geq 2-2\left(\frac{2}{3}\right)^{2-2}=0 .
$$

It remains to prove that the sum $\sum_{v=1}^{k-3}\left(k\left(\begin{array}{c}k-2 \\ v\end{array}\right)-\left(\begin{array}{c}k \\ v+1\end{array}\right)\right)$ is non-negative. Let us prove something slightly stronger, namely let us prove that $k\left(\begin{array}{c}k-2 \\ v\end{array}\right)-\left(\begin{array}{c}k \\ v+1\end{array}\right) \geq 0$ for $k \geq 2$ and $1 \leq v \leq k-3$. So note that

$$
\begin{aligned}
k\left(\begin{array}{c}
k-2 \\
v
\end{array}\right)-\left(\begin{array}{c}
k \\
v+1
\end{array}\right) & =\frac{k(k-2) !}{(v+1) !(k-1-v) !}((v+1)(k-v-1)-(k-1)) \\
& =\frac{k(k-2) !}{(v+1) !(k-1-v) !}(v(k-v-2)) \\
& \geq \frac{k(k-2) !}{(v+1) !(k-1-v) !}(v(k-(k-3)-2)) \\
& \geq 0
\end{aligned}
$$

Therefore, by Proposition 3.2 and Theorem 3.3, we have $p^{\prime \prime}\left(\operatorname{Id}_{\ell}+\operatorname{Rev}_{k}\right)=\mathfrak{U}_{\ell-1, \operatorname{Id}_{\ell}+\operatorname{Rev}_{k}}$. 

have

Now, by the definition of the Minimization Price Bound and a straightforward analysis argument, we

$$
\mathfrak{U}_{\ell-1, \operatorname{Id}_{\ell}+\operatorname{Rev}_{k}}=\min \left\{\sum_{j=1}^{\ell-1} x_{i}^{k}: \sum_{j=1}^{\ell-1} x_{j}=1 \text { and } \forall j \in[\ell-1], x_{j} \geq 0\right\}=\frac{1}{(\ell-1)^{k-1}} .
$$

\section{Concluding remarks}

As we mentioned in Section 2.1, Theorem 1.4 can be seen as a generalization of the following theorem.

Theorem 4.1 (Hästö (2002/03, Theorem 3.3)). If $\sigma \in \mathfrak{S}$ is a layered permutation whose layer sequence is $\left(\ell_{1}, \ell_{2}, \ldots, \ell_{k}\right)$, and $\ell_{1} \leq \ell_{2} \leq \cdots \leq \ell_{k}$ and $2^{\ell_{1}} \geq 1+k$, then $p(\sigma)=\mathfrak{L}_{k, \sigma}$.

As we also mentioned, Hästö presented the argument of the proof of Corollary 2.11 to give the following corollary.

Corollary 4.2 (Hästö (2002/03, Lemma 3.4)). If $\sigma \in \mathfrak{S}$ is a layered permutation with $k$ layers, all of which have lengths greater or equal to $\ell$ and we have $2^{\ell} \geq 1+k$, then $p(\sigma)=\mathfrak{L}_{k, \sigma}$.

The condition $2^{\ell} \geq 1+k$ of the above theorem can be seen as an analogous the condition $2^{a}-$ $a-1 \geq k$ of Theorem 1.4. The fact that $a+k$ is the number of layers of the permutation of block sequence $\left(\widehat{a}, \ell_{1}, \ell_{2}, \ldots, \ell_{k}\right)$ leads us to the following conjecture.

Conjecture 4.3. Let $\sigma \in \mathfrak{S}$ be a layered permutation with $k$ layers and a total of b blocks and suppose that every block of $\sigma$ has length greater or equal to $\ell$. If we have $2^{\ell} \geq 1+k$, then there exists a sequence $\left(\tau_{n}\right)_{n \in \mathbb{N}}$ of layered permutations of $b$ blocks such that $\left|\tau_{n}\right|<\left|\tau_{n+1}\right|$ for every $n \in \mathbb{N}$ and

$$
\lim _{n \rightarrow \infty} p\left(\sigma, \tau_{n}\right)=p(\sigma)
$$

Furthermore, Albert et al. (2002, Theorem 2.7) proved that if $\sigma \in \mathfrak{S}$ is a layered permutation with every layer of length at least 2 , then there is a sequence $\left(\tau_{n}\right)_{n \in \mathbb{N}}$ of layered permutations with $\left|\tau_{n}\right|<\left|\tau_{n+1}\right|$ for every $n \in \mathbb{N}$ and such that $\lim _{n \rightarrow \infty} p\left(\sigma, \tau_{n}\right)=p(\sigma)$ and the number of layers of the $\tau_{n}$ 's are uniformly bounded. For clarity, we state below a simplified version of their theorem.

Theorem 4.4 (Albert et al. (2002, Theorem 2.7)). If $\sigma \in \mathfrak{S}$ is a layered permutation with $k$ layers and every layer of length at least 2 , then there exists $K$ such that $p(\sigma)=\mathfrak{L}_{k+K, \sigma}$.

Later, Warren (2005) defined the smallest such $K$ to be the packing complexity of $\sigma$ (denoted $\kappa(\sigma)$ ).

The bound to $\kappa(\sigma)$ provided by Albert et al. (2002, Theorem 2.7), however, seems to be quite far from the real value.

Hästö's proof of Theorem 4.1 was generalized by Warren to improve this bound for the case when the layer sequence is non-decreasing.

Theorem 4.5 (Warren (2005, Theorem 3.3.7)). If $\sigma \in \mathfrak{S}$ is a layered permutation whose layer sequence is $\left(\ell_{1}, \ell_{2}, \ldots, \ell_{k}\right)$ and $2 \leq \ell_{1} \leq \ell_{2} \leq \cdots \leq \ell_{k}$, then $\kappa(\sigma) \leq \max \left\{(k-1) /\left(2^{\ell}-2\right), 0\right\}$.

A natural question would then be if the proof of Theorem 1.4 can be generalized to prove the following conjecture. 
Conjecture 4.6. If $\sigma$ has block sequence $\left(\widehat{a}, \ell_{1}, \ell_{2}, \ldots, \ell_{k}\right)$ with $2 \leq a \leq \ell_{1} \leq \ell_{2} \leq \cdots \leq \ell_{k}$, then there exists $K$ such that

$$
p(\sigma)=\mathfrak{L}_{K,[K] \backslash\{1\}, \sigma} .
$$

As several papers on permutation packing suggest, the family of layered permutations is much easier to work with than the general family of permutations due to Price Polynomials. Using the theory of permutons (i.e., limits of permutations, see Hoppen et al. (2013)), the fact that Price Bounds converge to $p(\sigma)$ can be seen as a topological property of permutons as we illustrate below.

Proposition 4.7. If $L_{k}$ is the family of permutons that can be obtained as limits of layered permutations with at most $k$ layers, then $\bigcup_{k \in \mathbb{N}} L_{k}$ is dense in the family of layered permutons (limits of layered permutations) with respect to the permuton topology (a sequence of permutons $\left(W_{n}\right)_{n \in \mathbb{N}}$ converges to $W$ if and only if $\lim _{n \rightarrow \infty} p\left(\sigma, W_{n}\right)=p(\sigma, W)$ for every $\left.\sigma \in \mathfrak{S}\right)$.

Proof : Either analogous to the proofs of Corollary 2.2 and Theorem 3.3 or by a standard diagonalization argument.

The proposition above by itself is not interesting, what is interesting is the fact that computing densities of layered permutations in permutons of $L_{k}$ is easy (yields Price Polynomials). In this light, Extended Price Polynomials can be seen simply as replacing $L_{k}$ with the family of permutons $B_{k}$ that can be obtained as limits of layered permutations with at most $k$ blocks instead of $k$ layers, which trivially preserves the density property (since $L_{k} \subset B_{k}$ ), but still yields a family in which densities of layered permutations are easy to compute.

As mentioned before, Price Polynomials are quite a useful tool when studying packing of layered permutations and there is still not an analogous tool for non-layered permutations, so the following natural question arises.

Question 4.8. Is there a family of permutons $F$ that is dense in the family of all permutons and is such that computing densities of permutations in permutons of $F$ is still easy?

Finally, Theorem 1.9 is a double-edged knife in the problem of minimization of monotone sequences, because, on the diagonal case (i.e., when $k=\ell$ ) it suggests that Conjecture 1.8 is true (since we have $\left.p^{\prime \prime}\left(\operatorname{Id}_{m+1}+\operatorname{Rev}_{m+1}\right)=1 / m^{m}\right)$. On the other hand, on the general case, it proves that

$$
p^{\prime \prime}\left(\operatorname{Id}_{3}+\operatorname{Rev}_{4}\right)=\frac{1}{2^{3}}>\frac{1}{3^{2}} \geq p^{\prime}\left(\operatorname{Id}_{3}+\operatorname{Rev}_{4}\right),
$$

which means that, when $k \neq \ell$, the problem restricted to the class of layered permutations is a distinct problem.

\section{References}

M. H. Albert, M. D. Atkinson, C. C. Handley, D. A. Holton, and W. Stromquist. On packing densities of permutations. Electron. J. Combin., 9(1):Research Paper 5, 20, 2002. ISSN 1077-8926. URL http://www.combinatorics.org/Volume_9/Abstracts/v9ilr5.html.

J. Balogh, P. Hu, B. Lidickỳ, O. Pikhurko, B. Udvari, and J. Volec. Minimum number of monotone subsequences of length 4 in permutations. 2013. URL http://homepages.warwick.ac.uk/ maskat/Papers/monoseq.pdf. 
P. Erdös and G. Szekeres. A combinatorial problem in geometry. Compositio Math., 2:463-470, 1935. ISSN 0010-437X. URL http://www.numdam.org/item?id=CM_1935_2_463_0.

P. A. Hästö. The packing density of other layered permutations. Electron. J. Combin., 9(2):Research paper 1, 16, 2002/03. ISSN 1077-8926. URL http: / /www.combinatorics.org/Volume_9/ Abstracts/v9i2r1.html. Permutation patterns (Otago, 2003).

C. Hoppen, Y. Kohayakawa, C. G. Moreira, B. Ráth, and R. Menezes Sampaio. Limits of permutation sequences. J. Combin. Theory Ser. B, 103(1):93-113, 2013. ISSN 0095-8956. doi: 10.1016/j.jctb.2012. 09.003. URL http://dx.doi.org/10.1016/j.jctb.2012.09.003.

J. S. Myers. The minimum number of monotone subsequences. Electron. J. Combin., 9(2):Research paper 4, 17 pp. (electronic), 2002/03. ISSN 1077-8926. URL http://www.combinatorics . org/Volume_9/Abstracts/v9i2r4.html. Permutation patterns (Otago, 2003).

A. L. Price. Packing densities of layered patterns. ProQuest LLC, Ann Arbor, MI, 1997. ISBN 978-0591-36275-6. URL http://gateway.proquest.com/openurl?url_ver=Z39. 88-2004\&rft_val_fmt=info: ofi/fmt:kev:mtx:dissertation\&res_dat=xri: pqdiss\&rft_dat=xri:pqdiss: 9727276 . Thesis (Ph.D.)-University of Pennsylvania.

A. A. Razborov. Flag algebras. J. Symbolic Logic, 72(4):1239-1282, 2007. ISSN 0022-4812. doi: 10.2178/jsl/1203350785. URL http://dx.doi.org/10.2178/jsl/1203350785.

W. Samotij and B. Sudakov. On the number of monotone sequences. J. Combin. Theory Ser. B, 115: 132-163, 2015. ISSN 0095-8956. doi: 10.1016/j.jctb.2015.05.008. URL http://dx.doi.org/ $10.1016 / j \cdot j$ jctb.2015.05.008.

D. Warren. Optimal packing behavior of some 2-block patterns. Ann. Comb., 8(3):355-367, 2004. ISSN 0218-0006. doi: 10.1007/s00026-004-0225-3. URL http://dx.doi.org/10.1007/ s00026-004-0225-3.

D. E. Warren. Optimizing the packing behavior of layered permutation patterns. ProQuest LLC, Ann Arbor, MI, 2005. ISBN 978-0542-17874-0. URL http://gateway proquest. com/openurl?url_ver=Z39.88-2004\&rft_val_fmt=info:ofi/fmt:kev:mtx: dissertation\&res_dat=xri:pqdiss\&rft_dat=xri:pqdiss: $3178050 . \quad$ Thesis (Ph.D.)-University of Florida. 